\title{
Associations of plasma very-long-chain SFA and the metabolic syndrome in adults ${ }^{*}$
}

\author{
Jing Zhao ${ }^{1}$, Xiaofan $\mathrm{Li}^{1}$, Xiang $\mathrm{Li}^{1}$, Qianqian $\mathrm{Chu}^{1}$, Yunhua Zhou ${ }^{2}, \mathrm{Zi} \mathrm{Li}^{1}$, Hong Zhang ${ }^{3}$,
} J. Thomas Brenna ${ }^{4,5}$, Yiqing Song ${ }^{6}$ and Ying $\mathrm{GaO}^{1 *}$

${ }^{1}$ Key Laboratory of Nutrition and Metabolism, Institute for Nutritional Sciences, Shanghai Institutes for Biological Sciences, Chinese Academy of Sciences, University of Chinese Academy of Sciences, 200031 Shanghai, People's Republic of China

${ }^{2}$ Department of Anatomy, Histology and Embryology, School of Basic Medical Sciences, Fudan University, 200032 Shanghai, People's Republic of China

${ }^{3}$ Institute of Biostatistics, Fudan University, 200433 Shanghai, People's Republic of China

${ }^{4}$ Dell Pediatric Research Institute, Dell Medical School, University of Texas at Austin, Austin, TX 78705, USA

${ }^{5}$ Division of Nutritional Sciences, Cornell University, Ithaca, NY 14853, USA

${ }^{6}$ Department of Epidemiology, Richard M. Fairbanks School of Public Health, Indiana University, Indianapolis, IN 46202, USA

(Submitted 23 January 2018 - Final revision received 15 June 2018 - Accepted 7 July 2018 - First published online 29 August 2018)

\section{Abstract}

Plasma levels of very-long-chain SFA (VLCSFA) are associated with the metabolic syndrome (MetS). However, the associations may vary by different biological activities of individual VLCSFA or population characteristics. We aimed to examine the associations of VLCSFA and MetS risk in Chinese adults. Totally, 2008 Chinese population aged 35-59 years were recruited and followed up from 2010 to 2012 . Baseline MetS status and plasma fatty acids data were available for 1729 individuals without serious diseases. Among 899 initially metabolically healthy individuals, we identified 212 incident MetS during the follow-up. Logistic regression analysis was used to estimate OR and 95\% CI. Crosssectionally, each VLCSFA was inversely associated with MetS risk; comparing with the lowest quartile, the multivariate-adjusted OR for the highest quartile were $0 \cdot 18$ (95\% CI 0.13, 0.25) for $\mathrm{C} 20: 0,0 \cdot 26$ (95\% CI 0.18, 0.35) for C22:0, 0.19 (95\% CI 0.13, 0.26) for C24:0 and 0.16 (0.11, $0 \cdot 22$ ) for total VLCSFA (all $P_{\text {for trend }}<0 \cdot 001$ ). The associations remained significant after further adjusting for C16:0, C18:0, C18:3n-3, C22: 6n-3,n-6 PUFA and MUFA, respectively. Based on follow-up data, C20:0 or C22:0 was also inversely associated with incident MetS risk. Among the five individual MetS components, higher levels of VLCSFA were most strongly inversely associated with elevated TAG $(\geq 1 \cdot 7 \mathrm{mmol} /$ 1). Plasma levels of VLCSFA were significantly and inversely associated with MetS risk and individual MetS components, especially TAG. Further studies are warranted to confirm the findings and explore underlying mechanisms.

\section{Key words: Cross-sectional studies: Hypertriacylglycerolaemia: Metabolic syndrome: SFA: Follow-up studies}

SFA have been linked to the development of metabolic disorders, including diabetes ${ }^{(1)}$, hyperlipidaemia ${ }^{(2)}$ and hypertension $^{(3)}$. The underlying mechanisms include activating NF- $\kappa \mathrm{B}$ to alter gene expression, serving as signal molecules and modu lating membrane fluidity ${ }^{(4,5)}$. The effect of straight chain SFA on metabolic process depends on the carbon chain length. Excess dietary palmitic acid (C16:0) impairs insulin sensitivity and increases blood pressure and lipids ${ }^{(6,7)}$. In contrast, very-longchain SFA (VLCSFA) with 20-24 carbon atoms may play a favourable role in metabolic disorders. Several epidemiological studies reported that higher circulating levels of VLCSFA were inversely associated with blood $\mathrm{TAG}^{(8)}$, insulin resistance ${ }^{(9)}$, incident diabetes ${ }^{(1,8)}$, arterial fibrillation ${ }^{(10)}, \mathrm{CHD}^{(11)}$, cardioembolic stroke ${ }^{(12)}$ and CVD mortality ${ }^{(13)}$.
Circulating VLCSFA are from minor dietary constituents and are also derived by endogenous synthesis from C16:0 or C18:0 as well as inter conversions among one another mediated by $\beta$-oxidation and elongation ${ }^{(14)}$. VLCSFA are found at significant quantities in a limited range of foods, including peanuts, lotus nuts and rapeseed/rapeseed oil ${ }^{(15)}$. In vivo, VLCSFA are major elements of ceramides and sphingomyelins, and the lower level of ceramides have been suggested to increase hepatocyte apoptosis and proliferation ${ }^{(16)}$, obesity risk ${ }^{(17)}$ and inflammatory response ${ }^{(17)}$ among many other structural roles in, for instance, the brain and $\operatorname{skin}^{(18,19)}$. Further studies have reported that some functions of ceramides are chain-length dependent ${ }^{(20)}$. For example, based on cell culture experiments, C24:0 promotes proliferation, whereas $\mathrm{C} 16: 0$ often has antiproliferative

Abbreviations: MetS, metabolic syndrome; VLCSFA, very-long-chain SFA; WC, waist circumference.

$\ddagger$ The original version of this article was published with an incorrect author's name. A notice detailing this has been published and the error rectified in the online PDF and HTML copies.

* Corresponding author: Y. Gao, email yinggao@sibs.ac.cn 
and pro-apoptotic effect ${ }^{(20)}$. Previous epidemiological studies showed that VLCSFA were inversely correlated with phospholipid C16:0 levels ${ }^{(8,10)}$, which suggested that VLCSFA and C16:0 might have competitive effect in some situation with opposite biological functions.

To our knowledge, no study to date has assessed the relation of VLCSFA to the metabolic syndrome (MetS) in a Chinese population. We aimed to explore the associations of plasma VLCSFA with prevalence of the MetS at baseline and risk of incident MetS of all initially metabolically healthy participants in a 2-year follow-up study. Further, we additionally evaluated the associations between VLCSFA with five MetS components.

\section{Methods}

\section{Study population}

The study population was from an investigation conducted by Chinese Centre for Disease Control and Prevention from April 2010 to December 2012 in Shunyi district of Beijing. Eligible participants had to live in the area for more than 1 year and had no plan to move within 1 year, no serious diseases of heart, lung, liver and kidney and no pregnancy. A total of 2008 participants aged between 35 and 59 years were recruited in Shunyi. All participants were interviewed in-person to collect information on demographic characteristics, history of diseases, medical conditions, current cigarette smoking status (at least one cigarette a day), alcohol drinking status (at least once a week during the past year), physical activity status (at least once a week, excluding walking and riding), agricultural working (typical agricultural workers), health education (conducted by local Centres for Disease Control) and other lifestyles. Height, weight, blood pressure and waist circumference (WC) were measured twice. Fasting venous blood was obtained for biochemical testing, including total cholesterol, HDL-cholesterol, TAG and plasma glucose. Plasma glucose was tested within $3 \mathrm{~h}$ and plasma lipids on the day of sampling. All biochemical testing was performed with an automatic biochemical analyser at Centre for Disease Control. Plasma was separated at $1500 \mathrm{~g} / \mathrm{min}$ centrifugation for $15 \mathrm{~min}$ and stored at $-80^{\circ} \mathrm{C}$. The above information and biosamples were collected a second time after 2 years.

Among the 1915 participants with available baseline fatty acid data, we excluded 185 participants with CHD, stroke, chronic respiratory diseases and malignant tumour at baseline; one participant with no MetS information was also excluded. Finally, 1729 participants were included in the current analyses. Among them, $81 \%$ had the 2-year follow-up data.

The study protocol was approved by the Ethical Review Committee of the National Centre for Chronic and Non-communicable Disease Control and Prevention, Chinese Centre for Disease Control and Prevention. All participants provided informed written consent. The trial was registered at chictr.org.cn as ChiCTREOC-17012759.

\section{Ascertainment of the metabolic syndrome}

The MetS was defined according to the updated National Cholesterol Education Program Adult Treatment Panel III criteria for Asian Americans ${ }^{(21)}$. Participants with any three of the five following items were identified as having the MetS: (1) WC $\geq 90 \mathrm{~cm}$ in men or $\geq 80 \mathrm{~cm}$ in women, (2) TAG $\geq 1.7 \mathrm{mmol} /$ 1, (3) HDL-cholesterol $\leq 1.03 \mathrm{mmol} / \mathrm{l}$ in men or $\leq 1.30 \mathrm{mmol} / \mathrm{l}$ in women, (4) blood pressure $\geq 130 / 85 \mathrm{mmHg}$ or current use of antihypertensive medications and (5) fasting plasma glucose $\geq 5.6 \mathrm{mmol} / \mathrm{l}$ or use of oral antidiabetic agents or insulin.

\section{Plasma fatty acids measurement}

GC/flame ionisation detector (FID) was used to measure plasma fatty acids profile ${ }^{(22,23)}$. Briefly, $100 \mu$ l of plasma with 1,2-dihenarachidoyl-sn-glycero-3-phosphocholine as internal standard was extracted in dichloromethane/methanol and the extracted lipids were reacted with methanol and sulphuric acid to yield fatty acid methyl ester (FAME). After methylation, FAME were extracted with $n$-hexane and redissolved in isooctane. Analysis was performed using an Agilent 6890 GC/FID equipped with a Supelco SP-2560 capillary column $(100 \mathrm{~m} \times 0.25 \mathrm{~mm}$ inside diameter $\times 0 \cdot 20 \mu \mathrm{m}$ thickness; Agilent Technologies Inc.). Data were expressed as weight percentage of total fatty acids. Samples were organised in batches of up to 20, which included two samples from a standard pool for quality control (QC). The CV of QC were 10.8\% for C20:0 (arachidic acid), 12.4\% for C22 : 0 (behenic acid) and 14.6\% for C24: 0 (lignoceric acid).

\section{Statistical analysis}

Wilcoxon rank-sum test for continuous variables and the $\chi^{2}$ test for categorical variables were used to examine the differences of basic characteristics between groups with the MetS and without the MetS. Spearman correlation was used to evaluate correlations of VLCSFA and other plasma fatty acids. Logistic regression analysis was applied to examine the associations of VLCSFA and risk of the MetS. VLCSFA were classified into four groups based on the quartiles of VLCSFA in participants without the MetS at baseline. Several models were fitted to examine the association of each VLCSFA and risk of the MetS: (1) model adjusted for age, sex and agricultural work (main model); (2) model adjusted for age, sex, agricultural work, education, smoking, alcohol drinking and physical activity (fully adjusted model) and (3) models additionally adjusted for the potential mediators based on fully adjusted model, including C16:0, C18: 0, C18:3n-3, C22:6n-3, n-6 PUFA and MUFA (exploration models). These selected fatty acids were significantly correlated with VLCSFA with the Spearman coefficients more than 0.25. As the fully adjusted models did not change the results, main model was used in the following analyses. The associations of VLCSFA and risk of incident MetS during 2-year follow-up were conducted only in participants without the MetS at baseline. Non-linear OR of the MetS was estimated by applying a restricted cubic spline regression model with three knots at the 5 th, 50th and 95th percentiles ${ }^{(24)}$. Multinomial logistic regression was used to estimate the associations of VLCSFA and risk of the MetS according to the number of MetS components at baseline. As the sample size in each group was limited, the VLCSFA levels were classified into high or low group based on the median of VLCSFA in subjects without the MetS. Low 
VLCSFA levels group were assigned as the reference. When the associations between VLCSFA and individual components of the MetS were evaluated, each of the other four components was adjusted in the models separately. To explore modification effects of some a priori risk factors on the association between VLCSFA and MetS risk, stratified analyses were conducted by age, sex, physical activity, agricultural working, smoking status and current alcohol drinking. Continuous variables were classified into high $v$. low levels based on the median levels in controls. The OR were expressed as the risk of the MetS for $1 \mathrm{SD}$ increase of VLCSFA. Likelihood ratio tests were conducted to examine interactions. All analyses were conducted with $\mathrm{R}$ software and SAS 9.3 software. All $P$ values were two-sided.

\section{Results}

A total of 1729 participants were included in this study; 565 $(32.7 \%)$ of these were identified as the MetS at baseline. Baseline characteristics of participants with or without the MetS were presented in Table 1. The two groups were similar in sex, smoking status, alcohol drinking and physical activity. Compared with participants without the MetS, the MetS participants were older and did less agricultural work. The MetS group had lower level of plasma C18:0, C20:0, C22:0, C24:0, C22:6n-3, C18:2n-6, C20:4n-6 and n-6 PUFA, but had higher C16:0, MUFA, C18:3n-3, C20:5n-3 and n-3 PUFA. Women and individuals without diabetes had higher levels VLCSFA (online Supplementary Tables S1a and S1b). In addition, higher VLCSFA were associated with lower BMI, lower TAG and higher HDL. Participants with higher levels of $\mathrm{C} 20: 0$ or $\mathrm{C} 22: 0$ tended to be non-smoker and non-alcohol drinker, do more agricultural work, have no hypertension and have lower WC and cholesterol (online Supplementary Table S1a). Furthermore, older ages were inversely related to higher $\mathrm{C} 20: 0$, but directly to higher C24:0 (online Supplementary Tables S1a and S1b). Participants with higher C24:0 were more likely to have hypertension (online Supplementary Table S1b).

All the three VLCSFA were significantly correlated with each another $\left(r_{\mathrm{s}} 0 \cdot 75,0 \cdot 23,0.21\right.$ for $\mathrm{C} 20: 0$ with $\mathrm{C} 22: 0, \mathrm{C} 24: 0$ and C22: 0 with C24: 0, online Supplementary Table S2). In the fully adjusted model, compared with the lowest quartile, the highest level of VLCSFA was associated with increased risk of the MetS; the multivariate-adjusted OR were $0 \cdot 18(95 \% \mathrm{CI} 0 \cdot 13,0 \cdot 25)$ for C20 : 0, $0 \cdot 26$ (95\% CI $0 \cdot 18,0 \cdot 35)$ for C22:0, $0 \cdot 19$ (95\% CI $0 \cdot 13$, $0 \cdot 26)$ for $\mathrm{C} 24: 0$ and $0.16(95 \%$ CI $0 \cdot 11,0.22)$ for total VLCSFA (Table 2). OR of the MetS were non-linear in C20:0, C22:0, C24:0 and total VLCSFA (Fig. 1, $P_{\text {non-linearity }}<0 \cdot 0001$ for $\mathrm{C} 20: 0$ and total VLCSFA, $P_{\text {non-linearity }}=0.002$ for $\mathrm{C} 22: 0$, $P_{\text {non-linearity }}=0.021$ for $\left.\mathrm{C} 24: 0\right)$. The VLCSFA were significantly correlated with C16:0, C18:0, MUFA, C18:3n-3, C22:6n-3 and $n$-6 PUFA with absolute $r_{\mathrm{s}}$ more than 0.25 (online Supplementary Table S2). Separately adjusted for these fatty acids did not affect the associations between VLCSFA and MetS risk (online Supplementary Table S3). In addition, the VLCSFA levels were inversely associated with the number of the MetS components (all the $P_{\text {trend }}<0.0001$, online Supplementary Table S4). In the multinomial logistic regression analysis, the
Table 1. Basic characteristics of subjects with and without the metabolic syndrome (MetS) at baseline

(Mean values and standard deviations; frequencies and percentages)

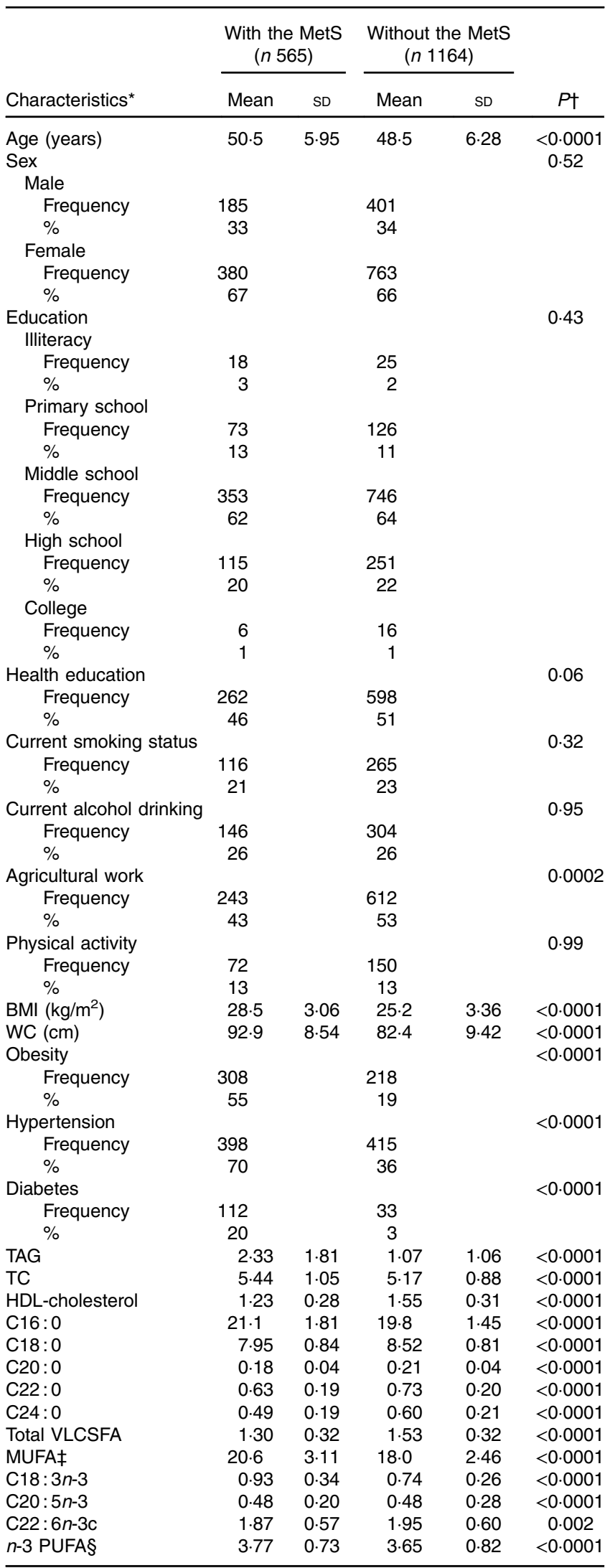


Table 1. Continued

\begin{tabular}{|c|c|c|c|c|c|}
\hline \multirow[b]{2}{*}{ Characteristics $^{*}$} & \multicolumn{2}{|c|}{$\begin{array}{l}\text { With the MetS } \\
\quad(n 565)\end{array}$} & \multicolumn{2}{|c|}{$\begin{array}{l}\text { Without the MetS } \\
\qquad(n \text { 1164) }\end{array}$} & \multirow[b]{2}{*}{$P \dagger$} \\
\hline & Mean & SD & Mean & SD & \\
\hline C18:2n-6 & 33.9 & 3.80 & $36 \cdot 1$ & 3.80 & $<0.0001$ \\
\hline $\mathrm{C} 20: 4 n-6$ & 7.32 & 1.79 & 8.55 & 1.73 & $<0.0001$ \\
\hline n-6 PUFAll & $44 \cdot 2$ & 3.99 & 47.6 & 3.06 & $<0.0001$ \\
\hline
\end{tabular}

WC, waist circumference; TC, total cholesterol; VLCSFA, very-long-chain SFA.

* Continuous variables were expressed as means and standard deviations and categorical variables were expressed as frequency (percentage among cases or controls).

$\dagger P$ values were calculated from Wilcoxon rank sum test for continuous variables and $X^{2}$ test for categorical variables.

¥ Includes C14:1n-5, C16:1n-7, C16:1n-9, C18:1n-7, C18:1n-9, C20:1n-9, C22: $1 n-9$ and $\mathrm{C} 24: 1 n-9$ in this study.

§ Includes C18:3n-3, C20:3n-3, C20:5n-3, C22:3n-3, C22:5n-3 and C22:6n-3. II Includes C18:2n-6, C18:3n-6, C20:2n-6, C20:3n-6, C20:4n-6, C22:2n-6, C22 : $4 n-6$ and C22: $5 n-6$ in this study.

associations of VLCSFA and MetS risk were stronger with the increase of the number of the MetS components (Table 3).

Among 899 subjects without the MetS at baseline, we identified 212 incident cases of the MetS during the 2-year follow-up. The level of C20:0, C22:0 and total VLCSFA was significantly associated with decreased incident MetS risk; comparing with the lowest quartile, the multivariate-adjusted $\mathrm{OR}$ for the highest quartile were $0.21(95 \% \mathrm{CI} 0.12,0.35)$ for $\mathrm{C} 20: 0,0.20(95 \% \mathrm{CI}$ $0 \cdot 11,0.34)$ for $\mathrm{C} 22: 0,0.75(95 \% \mathrm{CI} 0.44,1 \cdot 26)$ for $\mathrm{C} 24: 0$ and 0.28 (95\% CI 0.17, 0.46) for total VLCSFA (Table 4).

The associations between VLCSFA levels and each of five individual MetS components were analysed. Among the five MetS components, the associations were strongest for VLCSFA and elevated TAG (Q4 $v$. Q1: OR was $0 \cdot 11$ (95\% CI 0.07, 0.17) for $\mathrm{C} 20: 0,0 \cdot 10(95 \% \mathrm{CI} 0 \cdot 06,0 \cdot 16)$ for $\mathrm{C} 22: 0,0 \cdot 07$ (95\% CI
0.04, 0.12) for $\mathrm{C} 24: 0$ and 0.04 (95\% CI 0.02, 0.07) for total VLCSFA, $P_{\text {trend }}<0 \cdot 0001$, Fig. 2, online Supplementary Table S5).

We also explored effect modification on the associations of VLCSFA and MetS risk by several a priori risk factors, including age, sex, smoking status (yes and no), current alcohol drinking (yes and no), physical activity and agricultural working (Fig. 3). The inverse associations between $\mathrm{C} 20: 0$ and MetS risk were stronger in women than in men (OR $=0.37$ (95\% CI 0.31, 0.44) for women and OR=0.57 (95\% CI 0.46, 0.69) for men; $P$ for interaction $=0.002)$ and in non-alcohol drinkers than in current alcohol drinkers $(\mathrm{OR}=0.38(95 \% \mathrm{CI} 0.32,0.45)$ for non-alcohol drinkers and $\mathrm{OR}=0.61(95 \% \mathrm{CI} 0.49,0.75)$ for current alcohol drinkers; $P$ for interaction $=0 \cdot 0008)$ (Fig. 3).

\section{Discussion}

In a population of 1729 Chinese adults, we observed that plasma VLCSFA levels were significantly and inversely associated with prevalence and incidence of the MetS. Further adjustment for each of $\mathrm{C} 16: 0, \mathrm{C} 18: 0, \mathrm{C} 18: 3 n-3, \mathrm{C} 22: 6 n-3, n-6$ PUFA or MUFA did not negate the associations. The associations of VLCSFA and MetS risk became stronger with the increase of the number of the MetS components. Among the five individual MetS components, higher plasma VLCSFA were strongly associated with plasma TAG.

Our observations are generally in line with several previous studies that have reported the relationship between blood VLCSFA and various metabolic conditions in other populations ${ }^{(1,8,10-13,25)}$. Three prospective studies published recently showed that higher levels of three VLCSFA (C20:0, C22:0 and C24:0) were associated with lower risk of incident diabetes ${ }^{(1,8,25)}$. A study with 3179 participants from the Cardiovascular Health Study (CHS) reported that higher plasma phospholipid VLCSFA

Table 2. The associations of very-long-chain SFA (VLCSFA) and prevalence of the metabolic syndrome (MetS) at baseline* (Odds ratios and $95 \%$ confidence intervals)

\begin{tabular}{|c|c|c|c|c|c|c|c|c|}
\hline & \multirow[b]{2}{*}{ Quartile 1} & \multicolumn{2}{|c|}{ Quartile 2} & \multicolumn{2}{|c|}{ Quartile 3} & \multicolumn{2}{|c|}{ Quartile 4} & \multirow[b]{2}{*}{$P_{\text {trend }}$} \\
\hline & & OR & $95 \% \mathrm{Cl}$ & OR & $95 \% \mathrm{Cl}$ & OR & $95 \% \mathrm{Cl}$ & \\
\hline \multicolumn{9}{|l|}{ C20:0 } \\
\hline$n_{\text {MetS/non-MetS }}$ & $326 / 291$ & \multicolumn{2}{|c|}{$101 / 291$} & \multicolumn{2}{|c|}{$80 / 291$} & \multicolumn{2}{|c|}{$58 / 291$} & \\
\hline Model 1† & 1 & 0.30 & $0.23,0.40$ & 0.25 & $0.18,0.33$ & 0.18 & $0.13,0.25$ & $<0.0001$ \\
\hline Model 2‡ & 1 & 0.30 & $0.23,0.40$ & 0.25 & $0.18,0.33$ & 0.18 & $0.13,0.25$ & $<0.0001$ \\
\hline \multicolumn{9}{|l|}{$\mathrm{C} 22: 0$} \\
\hline$n_{\text {MetS/non-MetS }}$ & $248 / 291$ & \multicolumn{2}{|c|}{$153 / 291$} & \multicolumn{2}{|c|}{$98 / 291$} & \multicolumn{2}{|c|}{$66 / 291$} & \\
\hline Model 1† & 1 & 0.58 & $0.44,0.75$ & 0.38 & $0.28,0.50$ & $0 \cdot 26$ & $0.18,0.35$ & $<0.0001$ \\
\hline Model 2‡ & 1 & 0.58 & $0.44,0.75$ & 0.38 & $0.28,0.50$ & 0.26 & $0.18,0.35$ & $<0.0001$ \\
\hline \multicolumn{9}{|l|}{ C24:0 } \\
\hline$n_{\text {MetS/non-MetS }}$ & $260 / 291$ & \multicolumn{2}{|c|}{$160 / 291$} & \multicolumn{2}{|c|}{$90 / 291$} & \multicolumn{2}{|c|}{$55 / 291$} & \\
\hline Model 1† & 1 & 0.55 & $0.42,0.72$ & 0.30 & $0.22,0.40$ & 0.18 & $0.13,0.26$ & $<0.0001$ \\
\hline Model 2‡ & 1 & 0.55 & $0.42,0.71$ & 0.30 & $0.22,0.40$ & 0.19 & $0.13,0.26$ & $<0.0001$ \\
\hline \multicolumn{9}{|l|}{ Total VLCSFA } \\
\hline$n_{\text {MetS/non-MetS }}$ & $312 / 291$ & \multicolumn{2}{|c|}{$130 / 291$} & \multicolumn{2}{|c|}{$70 / 291$} & \multicolumn{2}{|c|}{$53 / 291$} & \\
\hline Model 1† & 1 & 0.38 & $0.29,0.50$ & 0.21 & $0.15,0.29$ & $0 \cdot 16$ & $0.11,0.22$ & $<0.0001$ \\
\hline Model 2‡ & 1 & 0.38 & $0.29,0.50$ & 0.21 & $0.15,0.29$ & $0 \cdot 16$ & $0.11,0.22$ & $<0.0001$ \\
\hline
\end{tabular}



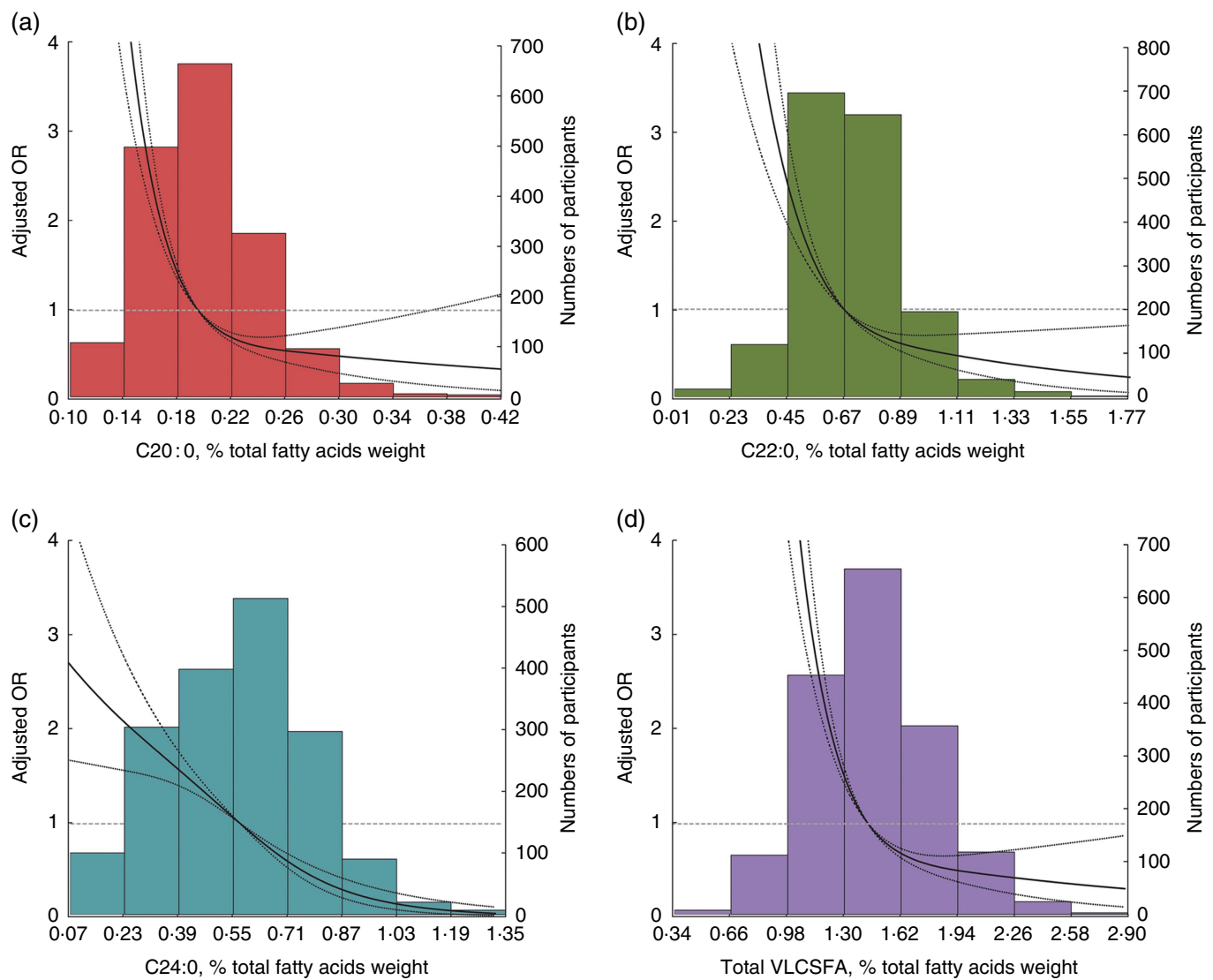

Fig. 1. Risk of the metabolic syndrome by very-long-chain SFA (VLCSFA) levels at baseline. Lines represent OR (95\% CI) based on restricted cubic splines for VLCSFA levels with knots at the 5th, 50th and 95th percentiles. OR were estimated using a logistic regression model after adjustment for age, sex and agricultural work; bars represent the numbers of participants. (a) C20:0 ( $\left.P_{\text {non-linearity }}<0.001\right)$, (b) $\mathrm{C} 22: 0\left(P_{\text {non-linearity }}=0.002\right)$, (c) $\mathrm{C} 24: 0\left(P_{\text {non-linearity }}=0.021\right)$ and $($ d $)$ total VLCSFA $\left(P_{\text {non-linearity }}<0.0001\right)$.

Table 3. Associations of very-long-chain SFA (VLCSFA) and risk of the metabolic syndrome (MS) according to the number of MS components at baseline*

(Odds ratios and $95 \%$ confidence intervals)

\begin{tabular}{lcccc}
\hline & $\mathrm{MS}=0$ & $\mathrm{MS}=3$ & $\mathrm{MS}=4$ & $\mathrm{MS}=5$ \\
\hline $20: 0$ & & & & \\
$n_{\text {high/low }}$ & $582 / 582$ & $80 / 251$ & $44 / 138$ & $14 / 38$ \\
OR & 1 & 0.33 & 0.33 & 0.39 \\
$95 \% \mathrm{Cl}$ & & $0.25,0.44$ & $0.23,0.47$ & $0.21,0.72$ \\
$22: 0$ & & & & \\
$n_{\text {high/low }}$ & $582 / 582$ & $81 / 250$ & $69 / 113$ & $14 / 38$ \\
OR & 1 & 0.32 & 0.61 & 0.36 \\
$95 \% \mathrm{Cl}$ & & $0.24,0.43$ & $0.44,0 \cdot 84$ & $0.19,0.67$ \\
$24: 0$ & & & & \\
$n_{\text {high/low }}$ & $582 / 582$ & $134 / 197$ & $8 / 174$ & $3 / 49$ \\
OR & 1 & 0.63 & 0.04 & 0.05 \\
$95 \% \mathrm{Cl}$ & & $0.49,0.81$ & $0.02,0.09$ & $0.02,0.18$ \\
Total VLCSFA & & & & \\
$n_{\text {high/low }}$ & $582 / 582$ & $97 / 234$ & $24 / 158$ & $2 / 50$ \\
OR & 1 & 0.40 & 0.15 & 0.04 \\
$95 \% \mathrm{Cl}$ & & $0.31,0.53$ & $0.09,0.23$ & $0.01,0.16$ \\
\hline
\end{tabular}

${ }^{*}$ Multinomial logistic regression was used to estimate the OR and $\mathrm{Cl}$. All the OR was adjusted for sex, age and agricultural work. As the sample size in each case group was limited, VLCSFA were classified into high/low group based on the median of VLCSFA in subjects without the metabolic syndrome. Low group was the reference.
Table 4. Associations of very-long-chain SFA (VLCSFA) and risk of incident metabolic syndrome (MetS) after 2 years* (Odds ratios and $95 \%$ confidence intervals)

\begin{tabular}{lccccc}
\hline & Quartile 1 & Quartile 2 & Quartile 3 & Quartile 4 & $P_{\text {trend }}$ \\
\hline C20:0 & & & & & \\
$n_{\text {MetS/non-MetS }}$ & $89 / 172$ & $62 / 171$ & $43 / 172$ & $18 / 172$ & \\
OR & 1 & 0.67 & 0.47 & 0.21 & $<0.001$ \\
$95 \%$ Cl & & $0.45,1.00$ & $0.30,0.72$ & $0.12,0.35$ & \\
C22:0 & & & & & \\
$n_{\text {MetS/non-MetS }}$ & $89 / 172$ & $65 / 171$ & $38 / 172$ & $20 / 172$ & \\
OR & 1 & 0.64 & 0.40 & 0.20 & $<0.001$ \\
$95 \%$ Cl & & $0.43,0.95$ & $0.25,0.62$ & $0.11,0.34$ & \\
C24:0 & & & & & \\
$n_{\text {MetS/non-MetS }}$ & $38 / 172$ & $87 / 171$ & $52 / 172$ & $35 / 172$ & \\
OR & 1 & 1.94 & 1.15 & 0.75 & 0.11 \\
$95 \%$ Cl & & $1.25,3.06$ & $0.71,1.87$ & $0.44,1 \cdot 26$ & \\
Total VLCSFA & & & & & \\
$n_{\text {MetS/non-MetS }}$ & $80 / 172$ & $62 / 171$ & $44 / 172$ & $26 / 172$ & \\
OR & 1 & 0.71 & 0.51 & 0.28 & $<0.001$ \\
$95 \%$ Cl & & $0.47,1.07$ & $0.33,0.78$ & $0.17,0.46$ & \\
\hline
\end{tabular}

* Only subjects without the MetS at baseline were included in the analysis. VLCSFA were classified into four group based on the quartiles of VLCSFA in subjects without the MetS: $0.18,0.21$ and 0.23 for C20:0; 0.60, 0.73 and 0.86 for C22:0; $0.46,0.63$ and 0.75 for C24:0; $1.32,1.51$ and 1.72 for total VLCSFA. Models were adjusted for sex, age and agricultural work. 


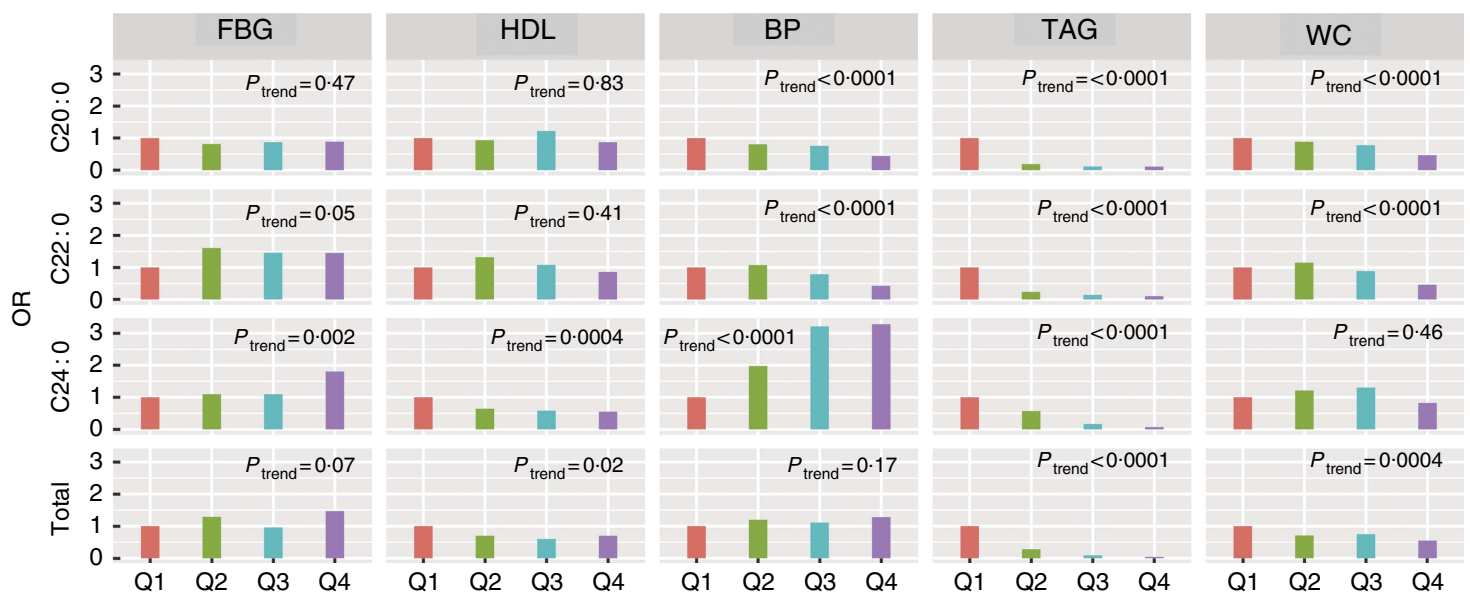

Fig. 2. Associations of very-long-chain SFA and risk of the metabolic syndrome components at baseline. Logistic regression was used to estimate the OR and Cl. All the OR were adjusted for age, sex and agricultural work. The five components were adjusted in the models, except for itself. The five components were classified into high/low group according to the cut-off in the metabolic syndrome definition. BP, blood pressure; FBG, fasting blood glucose; WC, waist circumference.

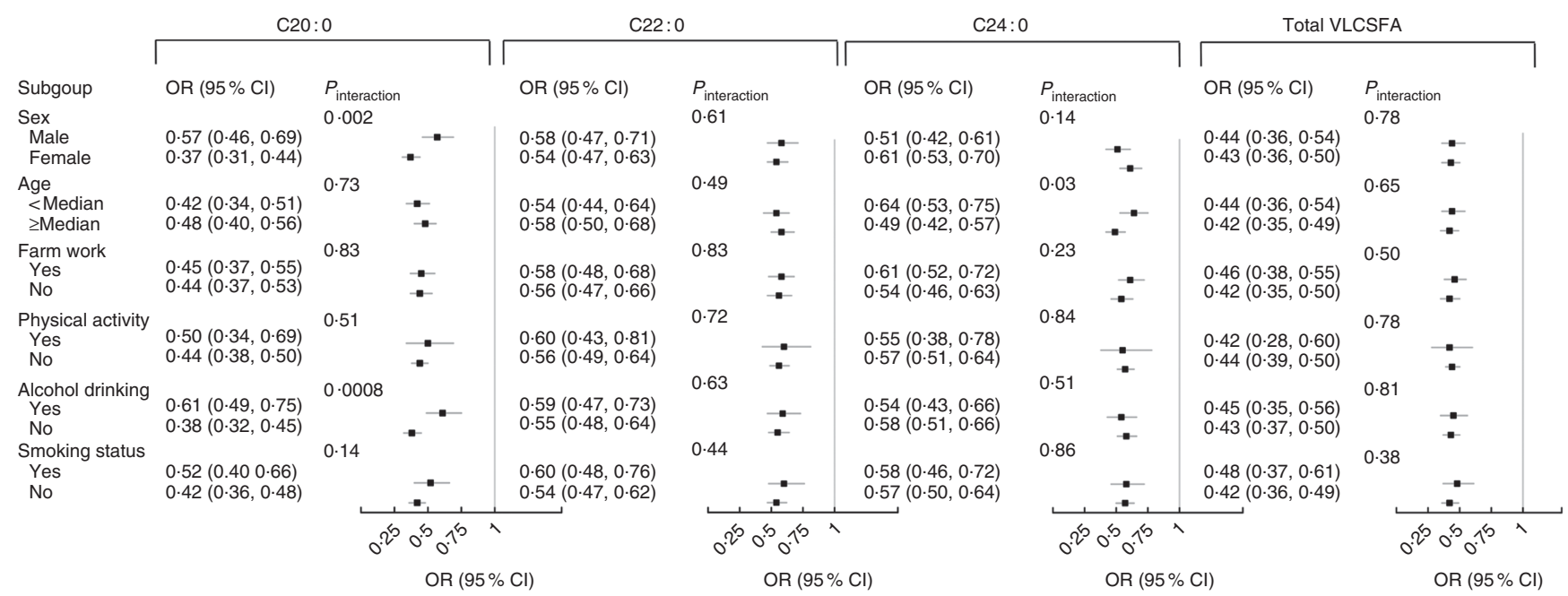

Fig. 3. Stratified analysis of the associations of very-long-chain SFA (VLCSFA) and risk of the metabolic syndrome at baseline. All the OR were adjusted for age, sex and agricultural work. The OR meant the risk of the metabolic syndrome for 1 SD increase of VLCSFA.

were cross-sectionally associated with lower blood TAG and $\mathrm{WC}^{(8)}$. In the Nurses' Health Study and Health Professionals Follow-Up Study, plasma VLCSFA were positively correlated with $\mathrm{HDL}^{(11)}$. Diabetes, WC, circulating TAG and HDL are components of the MetS. Consistently, we found inverse associations between plasma VLCSFA and MetS risk; TAG showed the strongest associations with VLCSFA. In addition, we found significant inverse associations of $\mathrm{C} 20: 0$ and $\mathrm{C} 22: 0$ with WC, and direct associations of C24:0 with HDL. However, we observed that C22:0 and C24: 0 were directly associated with fasting blood glucose, which was different from the results of above studies. If the covariate of TAG was excluded from the analyses model in our study, the associations of C22:0 and C24: 0 with fasting blood glucose were reversed. In the CHS study, VLCSFA were associated with lower risk of diabetes, but if blood TAG was adjusted in the model, the associations were not significant ${ }^{(8)}$. This suggested that the associations of VLCSFA with blood glucose might be mediated by lower TAG. Mechanistic studies are needed to confirm these results. Other recently published studies reported inverse associations of circulating levels of VLCSFA with atrial fibrillation ${ }^{(10)}$, cardioembolic stroke ${ }^{(12)}, \mathrm{CHD}^{(11)}$ and total mortality ${ }^{(13)}$. The MetS was a major intermediate risk factor of these diseases and may explain these associations ${ }^{(26-28)}$.

The biological functions of VLCSFA in vivo remain unclear. VLCSFA are major components of ceramides and sphingomyelins, which are key intermediates linking saturated fats and inflammatory cytokines to regulate cell function. Insulin resistance is the key feature of the MetS. VLCSFA are major components of ceramides and sphingomyelins. A large body of evidence from non-human experimental studies has supported a role of ceramides in insulin signalling and $\beta$-cell function as well as the development of diabetes ${ }^{(29)}$. Specifically, C16ceramide has been suggested to induce $\beta$-cell apoptosis, impair insulin signalling and reduce insulin synthesis, which can lead to insulin resistance ${ }^{(30,31)}$. In contrast, ceramides with VLCSFA appear to be protective. VLCSFA affect liver homoeostasis, 
myelin maintenance and anti-inflammatory response through inhibition of endogenous C16-ceramide synthesis ${ }^{(14,16)}$, but the biological pathways have not been fully understood and need to be investigated in future mechanistic studies. In addition, very-long-chain acyl-CoA synthetase may be up-regulated by $\operatorname{PPAR} \delta$ and evidence showed that PPAR $\delta$ played a role in fat metabolism to prevent obesity ${ }^{(32)}$

The sources of circulating VLCSFA include both exogenous diet and endogenous synthesis ${ }^{(14)}$. The VLCSFA are found in limited foods, including peanuts, lotus nuts and rapeseed/ rapeseed oil ${ }^{(15)}$. Several studies have shown that peanut intake increases blood $\mathrm{C} 22: 0$ and $\mathrm{C} 24: 0^{(8)}$. Interestingly, peanut butter consumption was significantly associated with a more favourable plasma lipid profile, including lower LDL-cholesterol, and total cholesterol in the Nurses' Health Study ${ }^{(33)}$. VLCSFA can be synthesised from C18:0 by elongases, especially elongation of very long chain fatty acids protein 1 (ELOVL1), which selectively elongates SFA with more than eighteen carbons ${ }^{(14)}$. Indeed, our data show that the fatty acids related to each other by a single round of elongation were highly correlated, suggesting that higher dietary intake resulted in higher levels of the elongation product: $18: 0 \rightarrow 20: 0$ and $20: 0 \rightarrow 22: 0$ (the Spearman coefficients were 0.48 for C18:0 and $\mathrm{C} 20: 0$, and 0.75 for $\mathrm{C} 20: 0$ and $\mathrm{C} 22: 0$ ). The ELOVL1 gene coding for the first committed step in elongation is regulated in concert with ceramide synthase 2 (CERS2), a key enzyme for C24 sphingolipid synthesis ${ }^{(34)}$. This regulation may ensure that the production of $\mathrm{C} 24-\mathrm{CoA}$ by elongation is coordinated with its utilisation. How the dietary intake and endogenous metabolism contribute to circulating levels of VLCSFA and subsequently affect the metabolic disorders requires further study.

We also explored the modifying effects by several a priori factors on the relation of VLCSFA to MetS risk. We found a stronger association between $\mathrm{C} 20$ :0 and MetS risk in subjects without alcohol drinking than those with current alcohol drinking. This phenomenon was consistent with the finding in the CHS study and our study that C20:0 was inversely correlated with the amount of alcohol drinks and current alcohol drinking status (yes/no) ${ }^{(8)}$. Heavy alcohol drinking is a risk factor for the MetS ${ }^{(35)}$ and may neutralise the beneficial effects of VLCSFA on the MetS. We also observed moderate interaction effects of sex on the association of $\mathrm{C} 20$ :0 with the MetS. In the CHS study and our study, women tended to have higher C20: $0^{(8)}$, which may explain our findings that the association of C20:0 and MetS risk was stronger in women than in men.

Our study is the first study to explore the associations of plasma VLCSFA and MetS risk in Chinese population. Our study has some limitations. First, the study design was initially crosssectional, but we confirmed the cross-sectional results among baseline non-MetS subjects with 2-year follow-up data. Second, the half-life of plasma fatty acids was short and it may not reflect long-term dietary pattern. The fatty acids of erythrocyte or fat adipose tissue were needed to confirm the associations of VLCSFA with MetS risk. Third, we analysed statistically plasma fatty acids in units of weight percentage as is reported in most studies $^{(8,11)}$, which is most useful in assessing whether fatty acid imbalance is related to metabolic status. Specific fatty acid concentration (e.g. $\mathrm{mg} / \mathrm{ml}$ plasma) is more appropriate to assess whether the mass of a specific circulating fatty acid is outside of a range that is needed to support the metabolism ${ }^{(36)}$. Best practices for the design, laboratory analysis, and reporting of trials involving fatty acids. Am J Clin Nutr in press). In addition, with the advancement of lipidomics technology, a detailed identification and quantification of VLCSFA in different lipid classes or tissues is of crucially helpful in understanding their roles in metabolic disorders. Unfortunately, we did not measure the fatty acids in different lipid classes (TAG, sphingomyelins, etc.). Fourth, no information on the lipid-lowering medication use was available. However, the hyperlipidaemia awareness rates $(8.3 \%)$, treatment rates $(7.0 \%)$, and detection rates $(26 \cdot 2 \%)$ were very low in China, especially in rural $\operatorname{areas}^{(37)}$, which suggests a possible mild lipid-lowering medication influence. Last but not least, compared with other fatty acids such as n-6 PUFA, C16:0 and MUFA, the proportion of VLSCFA was very low. However, after adjusting for each of these fatty acids separately, the association of VLCSFA and risk of the MetS remained statistically significant. Besides, we also adjusted for other potential confounders including lifestyle factors in the statistics model, but residual confounding by nonavailable or unknown factors is also possible, such as menopausal status of women or dietary VLCSFA.

In summary, we confirmed that higher levels of plasma VLCSFA are significantly associated with lower risk of the MetS in Chinese adults. The association of VLCSFA with blood TAG was stronger than other four MetS components. Further studies are warranted to explore the underlying mechanisms.

\section{Acknowledgements}

We thank Di Du from the Fudan University and Shuchun Lin from the Shanghai Institutes for Biological Sciences for their help in statistic consultation.

This work was supported by the National Key Research and Development Plan (Y. G., grant no. 2016YFD0400200) and the 100 talented plan of Chinese Academy of Sciences (Y. G.). This research received no specific grant from any funding agency, commercial or not-for-profit sectors. All funders had no role in the design, analysis or writing of this article.

Y. G. and J. Z. formulated the research questions and designed the study. J. Z., X. L., X. L., Q. C., Y. Z. and Z. L. carried it out. J. Z., X. L. and H. Z. analysed the data. J. Z., T. J. B., Y. S. and Y. G. wrote the article.

The authors declare that there are no conflicts of interest.

\section{Supplementary material}

To view supplementary material for this article, please visit https://doi.org/10.1017/S0007114518002106

\section{References}

1. Forouhi NG, Koulman A, Sharp SJ, et al. (2014) Differences in the prospective association between individual plasma phospholipid saturated fatty acids and incident type 2 diabetes: the epic-interact case-cohort study. Lancet Diabetes Endocrinol 2, 810-818. 
2. Siri-Tarino PW, Sun Q, Hu FB, et al. (2010) Saturated fat, carbohydrate, and cardiovascular disease. Am J Clin Nutr $\mathbf{9 1}$, 502-509.

3. Wang L, Manson JE, Forman JP, et al. (2010) Dietary fatty acids and the risk of hypertension in middle-aged and older women. Hypertension 56, 598-604.

4. Calder PC (2015) Functional roles of fatty acids and their effects on human health. JPEN J Parenter Enteral Nutr 39, 18S-32S.

5. Rocha DM, Caldas AP, Oliveira LL, et al. (2016) Saturated fatty acids trigger TLR4-mediated inflammatory response. Atherosclerosis 244, 211-215.

6. Mayneris-Perxachs J, Guerendiain M, Castellote AI, et al. (2014) Plasma fatty acid composition, estimated desaturase activities, and their relation with the metabolic syndrome in a population at high risk of cardiovascular disease. Clin Nutr 33, 90-97.

7. Kim OY, Lim HH, Lee MJ, et al. (2013) Association of fatty acid composition in serum phospholipids with metabolic syndrome and arterial stiffness. Nutr Metab Cardiovasc Dis 23, 366-374.

8. Lemaitre RN, Fretts AM, Sitlani CM, et al. (2015) Plasma phospholipid very-long-chain saturated fatty acids and incident diabetes in older adults: the cardiovascular health study. Am J Clin Nutr 101, 1047-1054.

9. Fernandez-Real JM, Broch M, Vendrell J, et al. (2003) Insulin resistance, inflammation, and serum fatty acid composition. Diabetes Care 26, 1362-1368.

10. Fretts AM, Mozaffarian D, Siscovick DS, et al. (2014) Plasma phospholipid saturated fatty acids and incident atrial fibrillation: the cardiovascular health study. J Am Heart Assoc 3, e000889.

11. Malik VS, Chiuve SE, Campos H, et al. (2015) Circulating verylong chain saturated fatty acids and incident coronary heart disease in US men and women. Circulation 132, 260-268.

12. Chung HK, Cho Y, Do HJ, et al. (2015) Plasma phospholipid arachidonic acid and lignoceric acid are associated with the risk of cardioembolic stroke. Nutr Res 35, 1001-1008.

13. Fretts AM, Mozaffarian D, Siscovick DS, et al. (2016) Associations of plasma phospholipid SFAs with total and cause-specific mortality in older adults differ according to SFA chain length. J Nutr 146, 298-305.

14. Kihara A (2012) Very long-chain fatty acids: elongation, physiology and related disorders. J Biochem 152, 387-395.

15. Yang Y, Wang G \& Pan X (2009) China Food Composition, 257-303. Beijing: Peking University Medical Press.

16. Pewzner-Jung Y, Brenner O, Braun S, et al. (2010) A critical role for ceramide synthase 2 in liver homeostasis: II. Insights into molecular changes leading to hepatopathy. J Biol Chem 285, 10911-10923.

17. Samad F, Badeanlou L, Shah C, et al. (2011) Adipose tissue and ceramide biosynthesis in the pathogenesis of obesity. $A d v$ Exp Med Biol 721, 67-86.

18. Mielke MM, Bandaru VV, Haughey NJ, et al. (2012) Serum ceramides increase the risk of Alzheimer disease: the women's health and aging study II. Neurology 79, 633-641.

19. Choi MJ \& Maibach HI (2005) Role of ceramides in barrier function of healthy and diseased skin. Am J Clin Dermatol 6, $215-223$.
20. Grosch S, Schiffmann S \& Geisslinger G (2012) Chain lengthspecific properties of ceramides. Prog Lipid Res 51, 50-62.

21. Grundy SM, Cleeman JI, Daniels SR, et al. (2005) Diagnosis and management of the metabolic syndrome: an American Heart Association/National Heart, Lung, and Blood Institute scientific statement. Circulation 112, 2735-2752.

22. Sun Q, Ma J, Campos H, et al. (2007) Comparison between plasma and erythrocyte fatty acid content as biomarkers of fatty acid intake in US women. Am J Clin Nutr 86, 74-81.

23. Han X, Rozen S, Boyle SH, et al. (2011) Metabolomics in early Alzheimer's disease: identification of altered plasma sphingolipidome using shotgun lipidomics. PLOS ONE $\mathbf{6}$, e21643.

24. Desquilbet L \& Mariotti F (2010) Dose-response analyses using restricted cubic spline functions in public health research. Stat Med 29, 1037-1057.

25. Alhazmi A, Stojanovski E, Garg ML, et al. (2014) Fasting whole blood fatty acid profile and risk of type 2 diabetes in adults: a nested case-control study. PLOS ONE 9, e97001.

26. Hata J, Doi Y, Ninomiya T, et al. (2010) The effect of metabolic syndrome defined by various criteria on the development of ischemic stroke subtypes in a general Japanese population. Atherosclerosis 210, 249-255.

27. Nystrom PK, Carlsson AC, Leander K, et al. (2015) Obesity, metabolic syndrome and risk of atrial fibrillation: a Swedish, prospective cohort study. PLOS ONE 10, e0127111.

28. Janczura M, Bochenek G, Nowobilski R, et al. (2015) The relationship of metabolic syndrome with stress, coronary heart disease and pulmonary function - an occupational cohortbased study. PLOS ONE 10, e0133750.

29. Chavez JA \& Summers SA (2012) A ceramide-centric view of insulin resistance. Cell Metab 15, 585-594.

30. Galadari S, Rahman A, Pallichankandy S, et al. (2013) Role of ceramide in diabetes mellitus: evidence and mechanisms. Lipids Health Dis 12, 98.

31. Summers SA (2006) Ceramides in insulin resistance and lipotoxicity. Prog Lipid Res 45, 42-72.

32. Wang Y-X, Lee C-H, Tiep S, et al. (2003) Peroxisomeproliferator-activated receptor $\delta$ activates fat metabolism to prevent obesity. Cell 113, 159-170.

33. Li TY, Brennan AM, Wedick NM, et al. (2009) Regular consumption of nuts is associated with a lower risk of cardiovascular disease in women with type 2 diabetes. J Nutr 139, $1333-1338$.

34. Ohno Y, Suto S, Yamanaka M, et al. (2010) Elovl1 production of c24 acyl-coas is linked to c24 sphingolipid synthesis. Proc Natl Acad Sci U S A 107, 18439-18444.

35. Baik I \& Shin C (2008) Prospective study of alcohol consumption and metabolic syndrome. Am J Clin Nutr 87, $1455-1463$.

36. Brenna JT, Plourde M, Stark KD, et al. (2018) Best practices for the design, laboratory analysis, and reporting of trials involving fatty acids. Am J Clin Nutr (epublication ahead of print version 21 June 2018).

37. Chang J \& Wang Y (2016) Chinese Residents of Nutrition and Health Condition Monitoring 2012-2013, pp. 108-128. Beijing: Peking Medical University Press. 